\title{
Residual White Space Distribution-Based Opportunistic Channel Access for Cognitive Radio Enabled Devices
}

\author{
Manuj Sharma and Anirudha Sahoo \\ Dept. of Computer Science and Engineering \\ Indian Institute of Technology Bombay \\ Mumbai, India \\ manuj@it.iitb.ac.in, sahoo@it.iitb.ac.in
}

\begin{abstract}
We describe an opportunistic channel access scheme for cognitive radio-enabled secondary nodes $(\mathrm{SNs})$. The proposed scheme uses the residual channel idle time distribution to estimate the transmission duration in the remaining idle time, subject to an acceptable Primary User (PU) interference constraint. The SN then transmits the frames within the estimated duration without further sensing the channel, which reduces sensing overhead. The scheme does not require the SN to continuously sense the channel to keep track of the start of the idle period, thereby conserving energy.

Categories and Subject Descriptors:C.2.5 [Local and Wide-Area Networks]: Access schemes

General Terms: Theory, Performance
\end{abstract}

\section{INTRODUCTION}

Opportunistic Spectrum Access (OSA) by cognitive radioenabled secondary devices has emerged as one of the promising approaches to increase utilization of underutilized licensed spectrum bands. However, OSA approach requires that the secondary users should not violate any acceptable interference bounds specified by the licensed (primary) users. In this abstract, we describe an opportunistic channel access scheme for energy-constrained secondary devices. The key contribution of the reported work is derivation and application of residual idle time distribution of the channel for opportunistic channel access, which reduces the channel sensing operations and consequently, the energy consumption due to channel sensing.

\section{MOTIVATION AND CHALLENGES}

The primary motivation to use OSA approach is to increase the utilization of idle periods (usually referred as white spaces or holes) in various spectrum bands by allowing secondary devices to use these white spaces for their communication. The OSA scheme strives to maximize the secondary node's throughput, without violating acceptable interference bound set by the primary user.

The main challenges involved in devising an OSA scheme for energy-constrained secondary devices are as follows. First, the secondary nodes should be able to identify the white spaces in the spectrum and utilize them. However, they should be able to vacate the spectrum when primary reap-

Copyright is held by the author/owner(s).

SIGCOMM'10, August 30-September 3, 2010, New Delhi, India. ACM 978-1-4503-0201-2/10/08. pears for transmission. Hence, the challenge is to maximize the white space utilization and secondary node throughput without violating the PU interference bound. Second, the OSA scheme should minimize the channel sensing (and therefore, the energy consumed in the sensing operations) by the secondary node.

\section{CURRENT SOLUTIONS AND PROBLEM STATEMENT}

Current schemes, such as [1], which use channel idle time distribution for opportunistic access, assume that either the start of idle period (white space) is known to the Secondary Node (SN), or the secondary network transmission is slotted and the SN senses the channel at the beginning of each slot. Keeping track of start of each idle period requires the SN to continuously sense the channel, which is not suitable for energy-constrained secondary devices.

So, we devise a channel access strategy for energy constrained, cognitive radio-enabled secondary devices to opportunistically use a wireless channel when it is found to be idle. The scheme should ensure that the SN transmissions do not cause interference to any PU beyond a certain limit and that channel sensing (to determine idle periods) is minimized to conserve energy.

\section{OUR PROPOSED SCHEME}

We assume that the SN knows the channel idle and busy time distributions due to PU activity. In the proposed scheme, the SN computes the residual idle time distribution of the channel. It senses the channel only at time instant when one of more data frames arrive (from upper layer) for transmission, thereby reducing the channel sensing operations. If the channel is idle, it uses the residual idle time distribution for opportunistic transmissions such that these transmissions do not cause interference to any PU beyond a certain limit $\eta$. The proposed theory is general in nature and should work with any distribution fitted to the real channel idle time traces. We have used 2-Erlang and Uniform distributions as examples. To the best of our knowledge, opportunistic spectrum access in cognitive radio networks using residual idle time distribution has not been reported earlier in the literature.

\subsection{Access Strategy}

Let $I$ and $B$ represent the random variables denoting channel idle and busy time values, and $F_{I}$ and $F_{B}$ represent respectively the channel idle and busy time distribu- 
tions with known parameters. Let $F_{R I}$ represent the residual idle time (or residual white space) distribution function, which is computed by the $\mathrm{SN}$ as follows [3, pp. 331]:

$$
F_{R I}(y)=\frac{\int_{0}^{y}\left(1-F_{I}(z)\right) \mathrm{d} z}{E[I]}
$$

Here $E[I]$ denotes the mean channel idle time and $F_{R I}(y)$ denotes the probability that the residual white space is less than $y$. On sensing the channel idle, the SN computes the transmission duration $y$ in the remaining white space so that the following constraint is satisfied:

$$
F_{R I}(y) \leq \eta
$$

The maximum value of $y$ (which we denote as $y_{\max }$ ) for which the above inequality is satisfied, is taken as the transmission duration by the $\mathrm{SN}$ in the remaining white space.

\subsection{Average Secondary Node Throughput per White Space}

For a given $y_{\max }$ value, which is computed by solving (2), the average $\mathrm{SN}$ throughput per white space is given as:

$$
A T H P W S=\frac{\left[y_{\max }\left(1-F_{R I}\left(y_{\max }\right)\right)+\int_{q=0}^{y_{\max }} q f_{R I}(q) \mathrm{d} q\right] * R}{S}
$$

Here, integral variable $q$ denotes the values that residual idle time random variable $R I$ takes, $R$ is the data transmission rate of the wireless channel (in bits per second), and $S$ is the size of secondary node frame (in bits).

If we assume that the channel idle time values are 2-Erlang Distributed (with parameter $\lambda_{i}$ ), the expression to compute average SN throughput per white space can be written as (see [2] for derivations):

$$
A T H P W S=\frac{\left[\frac{3}{2 \lambda_{i}}-e^{-\lambda_{i} y_{\max }}\left(\frac{y_{\max }}{2}+\frac{3}{2 \lambda_{i}}\right)\right] * R}{S}
$$

\subsection{Primary User Interference Probability due to Secondary Transmissions}

For a given $y_{\max }$ value, the $\mathrm{PU}$ interference probability due to $\mathrm{SN}$ transmissions is given as:

$$
P U I P=\int_{q=0}^{y_{\max }} f_{R I}(q) \mathrm{d} q
$$

For 2-Erlang distributed channel idle time values, this metric is written as follows [2]:

$$
P U I P=1-\frac{e^{-\lambda_{i} y_{\max }}}{2}\left(2+\lambda_{i} \cdot y_{\max }\right)
$$

\section{SUMMARY OF RESULTS}

The main results obtained using simulations are as follows. First, the larger the value of $\eta$ (i.e. larger acceptable PU interference), the higher the average throughput of SN (in frames/sec) per white space (see Figure 1). This is so because the proposed theory predict larger transmission times (i.e. larger $y_{\max }$ values), resulting in aggressive transmission by $\mathrm{SN}$ in each white space. However, such aggressive transmission by the SN leads to higher PU interference. For

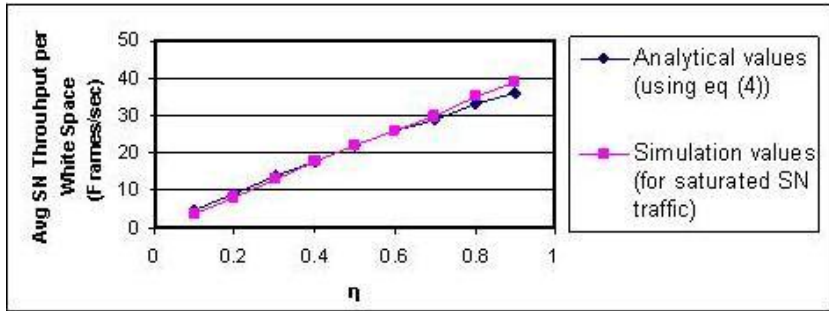

Figure 1: Avg SN Throughput per White Space $\left(\lambda_{i}=\right.$ $\left.200 \mathrm{sec}^{-1}\right)$; SN Frame Size $=\mathbf{2 0 4 8}$ bits; Channel rate $=$ 11 Mbps

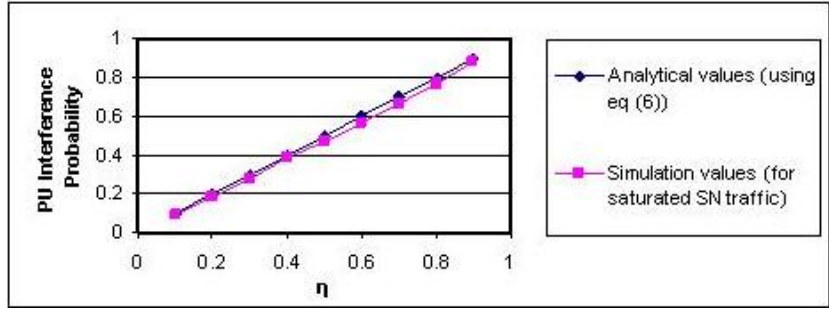

Figure 2: PU Interference Probability $\left(\lambda_{i}=200 \mathrm{sec}^{-1}\right)$; SN Frame Size $=2048$ bits; Channel rate $=11 \mathrm{Mbps}$

smaller values of $\eta$, the estimated values of $y_{\max }$, and consequently the SN transmissions, are conservative (so that it can meet the low interference requirement of the Primary Network), which leads to low SN throughput per white space but low interference to the PU. Second, the analytically computed value PUIP serves as an upper bound for probability of interference to PU's transmission (due to secondary transmissions) (see Figure 2). Moreover, PUIP for a given $\eta$ is always less than or equal to the corresponding $\eta$. The proposed theory ensures that the acceptable PU interference bound is never violated due to SN transmissions. The analytically computed $A T H P W S$ values in Figure 1 matches reasonably well with the simulation-obtained values for saturated SN traffic. For additional results, please refer to [2].

As part of our future work, we plan to do sensitivity analysis of the proposed scheme with respect to the distribution parameters, compare the scheme with the other proposed scheme (such as [1]) and other PU traffic profiles, and extend the scheme for multi SN, multi channel scenarios.

\section{REFERENCES}

[1] A. Plummer, M. Taghizadeh, and S. Biswas. Measurement Based Capacity Scavenging via Whitespace Modeling in Wireless Networks. In Proc. of IEEE GLOBECOM, 2009.

[2] M. Sharma and A. Sahoo. Residual White Space Distribution Based Opportunistic Channel Access Scheme for Cognitive Radio Systems. Technical report, Indian Institute of Technology (IIT) Bombay, June 2010. Available Online:

http://www.cse.iitb.ac.in/internal/techreports/reports/TRCSE-2010-30.pdf

[3] K. S. Trivedi. Probability and Statistics with Reliability, Queuing and Computer Science Applications. John Wiley and Sons, Inc., 2nd edition, 2002. 